PROCEEDINGS OF THE

AMERICAN MATHEMATICAL SOCIETY

Volume 135, Number 2, February 2007, Pages 453-460

S 0002-9939(06)08485-1

Article electronically published on August 8, 2006

\title{
ON SUBSYMMETRIC BASES IN FRÉCHET SPACES
}

\author{
WIESŁAW ŚLIWA
}

(Communicated by N. Tomczak-Jaegermann)

\begin{abstract}
It is proved that any non-normable Fréchet space with a semisymmetric absolute basis is isomorphic to the space $\omega$ of all scalar sequences. A similar result is shown for quasi-homogeneous absolute bases. It is also proved that any nuclear Fréchet space with a semi-subsymmetric basis is isomorphic to $\omega$.
\end{abstract}

\section{INTRODUCTION}

Let $E$ be a Fréchet space (i.e. a metrizable lcs over the field $\mathbb{K}$ of real or complex numbers) with a base $\left(p_{k}\right)$ of continuous seminorms. A basis $\left(e_{n}\right)$ in $E$, with the sequence $\left(f_{n}\right)$ of coefficient functionals, is said to be absolute if

$$
\forall k \in \mathbb{N} \forall x \in E: \sum_{n=1}^{\infty}\left|f_{n}(x)\right| p_{k}\left(e_{n}\right)<\infty .
$$

By the Dynin-Mitiagin theorem any basis in a nuclear Fréchet space is absolute.

A basis $\left(e_{n}\right)$ in $E$ is $\left(s e m i\right.$-) symmetric if any permutation $\left(e_{\pi(n)}\right)$ of $\left(e_{n}\right)$ is a basis in $E$ (semi-)equivalent to $\left(e_{n}\right)$. We say that a basis $\left(e_{n}\right)$ in $E$ is (semi-)subsymmetric if any permutation $\left(e_{\pi(n)}\right)$ of $\left(e_{n}\right)$ is a basis in $E$ (semi-)equivalent to some subsequence $\left(e_{k_{n}}\right)$ of $\left(e_{n}\right)$. (Two sequences $\left(x_{n}\right)$ in an lcs $X$ and $\left(y_{n}\right)$ in an lcs $Y$ are equivalent if there exists an isomorphism $T$ between their linear spans such that $T x_{n}=y_{n}, n \in \mathbb{N}$, and semi-equivalent if for some $\left(\alpha_{n}\right) \subset(\mathbb{K} \backslash\{0\})$ the sequences $\left(x_{n}\right),\left(\alpha_{n} y_{n}\right)$ are equivalent.)

Symmetric bases were studied in Banach spaces by Singer [8] and in locally convex spaces (lcs) by Garling [4, Ruckle [6], 7], Cac [3] and Terzioğlu [9].

Every basis in $\omega$ is symmetric because any two bases in $\omega$ are equivalent [2]. Terzioğlu [9] proved that any nuclear Fréchet space with a symmetric basis is isomorphic to $\omega$. (His definition of a symmetric basis is equivalent to our definition which appears in 7 .)

In this paper we show that any non-normable Fréchet space with a semi-symmetric absolute basis is isomorphic to $\omega$ (Theorem 2). We also prove that any FréchetSchwartz space with a semi-subsymmetric absolute basis is isomorphic to $\omega$ (Theorem 3). It follows that any nuclear Fréchet space with a semi-subsymmetric basis (in particular, with a subsymmetric basis) is isomorphic to $\omega$ (Corollary 4 ).

Received by the editors October 6, 2004 and, in revised form, September 6, 2005.

2000 Mathematics Subject Classification. Primary 46A35.

Key words and phrases. Symmetric, semi-symmetric, semi-subsymmetric, quasi-homogeneous bases.

(C)2006 American Mathematical Society 
Next, we construct an uncountable family of mutually non-quasi-equivalent subsymmetric absolute bases in Fréchet spaces (Proposition 5). (Two sequences $\left(x_{n}\right)$ in an lcs $X$ and $\left(y_{n}\right)$ in an lcs $Y$ are quasi-equivalent if for some permutation $\pi$ of $\mathbb{N}$ the sequences $\left(x_{n}\right),\left(y_{\pi(n)}\right)$ are semi-equivalent.)

We say that a basis $\left(e_{n}\right)$ in $E$ is (quasi-)homogeneous if any subsequence $\left(e_{k_{n}}\right)$ of $\left(e_{n}\right)$ is (quasi-)equivalent to $\left(e_{n}\right)$. (Homogeneous bases in Banach spaces are known as "subsymmetric bases" 8 .)

We show that any non-normable Fréchet space with a quasi-homogeneous absolute basis is isomorphic to $\omega$ (Theorem 6 ). It follows that any nuclear Fréchet space with a quasi-homogeneous basis (in particular, with a homogeneous basis) is isomorphic to $\omega$ (Corollary 7 ).

\section{Preliminaries}

The linear span of a subset $A$ of a linear space $E$ is denoted by $\operatorname{lin} A$.

A non-decreasing sequence $\left(p_{k}\right)$ of continuous seminorms on an lcs $E$ is a base of continuous seminorms on $E$ if for every continuous seminorm $p$ on $E$ there is $k \in \mathbb{N}$ with $p \leq p_{k}$. Any metrizable lcs $E$ possesses a base $\left(p_{k}\right)$ of continuous seminorms.

A Fréchet space $F$ with a base $\left(q_{k}\right)$ of continuous seminorms is isomorphic to $\omega$ if and only if for any $k \in \mathbb{N}$ the quotient space $\left(F / \operatorname{ker} q_{k}\right)$ is finite-dimensional [1].

A sequence $\left(x_{n}\right)$ in an lcs $E$ is a basis in $E$ if each $x \in E$ can be written uniquely as $x=\sum_{n=1}^{\infty} \alpha_{n} x_{n}$ with $\left(\alpha_{n}\right) \subset \mathbb{K}$. If additionally the coefficient functionals $f_{n}: E \rightarrow \mathbb{K}, x \rightarrow \alpha_{n}(n \in \mathbb{N})$ are continuous, then $\left(x_{n}\right)$ is a Schauder basis in $E$.

Any basis in a Fréchet space is a Schauder basis.

Let $\left(x_{n}\right)$ be a basis in a Fréchet space $X$ and $\left(y_{n}\right)$ a basis in a Fréchet space $Y$. Then the following conditions are equivalent:

(1) $\left(x_{n}\right)$ is equivalent to $\left(y_{n}\right)$;

(2) for any $\left(\alpha_{n}\right) \subset \mathbb{K}$ the series $\sum_{n=1}^{\infty} \alpha_{n} x_{n}$ is convergent in $X$ if and only if the series $\sum_{n=1}^{\infty} \alpha_{n} y_{n}$ is convergent in $Y$.

If a sequence $\left(x_{n}\right)$ in a Fréchet space $X$ is quasi-equivalent to a sequence $\left(y_{n}\right)$ in a Fréchet space $Y$, then the closed linear spans of $\left(x_{n}\right)$ and $\left(y_{n}\right)$ are isomorphic.

Let $E$ be a Fréchet space with a base $\left(p_{k}\right)$ of continuous seminorms and let $\left(e_{n}\right)$ be an absolute basis in $E$ with the sequence $\left(f_{n}\right)$ of coefficient functionals. Then we have the following:

(a) For any $M \subset \mathbb{N},\left(e_{n}\right)_{n \in M}$ is an absolute basis in the closed linear span $E_{M}$ of $\left(e_{n}\right)_{n \in M}$ and $E$ is isomorphic to the product $E_{M} \times E_{\mathbb{N} \backslash M}$.

(b) The seminorms $p_{k}^{*}, k \in \mathbb{N}$, defined by

$$
p_{k}^{*}(x)=\sum_{n=1}^{\infty}\left|f_{n}(x)\right| p_{k}\left(e_{n}\right), x \in E
$$

are continuous on $E$ and $\left(p_{k}^{*}\right)$ is a base of continuous seminorms on $E$.

(c) $E$ is a nuclear space (respectively, a Schwartz space) if and only if for any $i \in \mathbb{N}$ there exists $j>i$ such that $\sum_{n=1}^{\infty}\left[p_{i}\left(e_{n}\right) / p_{j}\left(e_{n}\right)\right]<\infty$ (respectively, $\left.\left[p_{i}\left(e_{n}\right) / p_{j}\left(e_{n}\right)\right] \rightarrow_{n} 0\right)($ we agree $[0 / 0]=0)[5]$.

Let $B=\left(b_{n, k}\right)$ be an infinite matrix consisting of positive real numbers and satisfying the condition $\forall k, n \in \mathbb{N}: b_{n, k} \leq b_{n, k+1}$. The Köthe space associated with 
the matrix $B$ is the Fréchet space

$$
K(B)=\left\{\left(\alpha_{n}\right) \subset \mathbb{K}: \sum_{n=1}^{\infty}\left|\alpha_{n}\right| b_{n, k}<\infty \text { for all } k \in \mathbb{N}\right\}
$$

with the standard base $\left(p_{k}\right)$ of continuous norms: $p_{k}\left(\left(\alpha_{n}\right)\right)=\sum_{n=1}^{\infty} k\left|\alpha_{n}\right| b_{n, k}, k \in$ $\mathbb{N}$. The sequence $\left(e_{n}\right)$ of coordinate vectors is an absolute basis in $K(B)$.

\section{RESUlts}

We will need the following lemma.

Lemma 1. Assume that a sequence $\left(x_{n}\right)$ in a metrizable lcs $X$ is semi-equivalent to a sequence $\left(y_{n}\right)$ in a metrizable lcs $Y$. Let $\left(p_{k}\right)$ and $\left(q_{k}\right)$ be bases of continuous seminorms on $X$ and $Y$, respectively. Then there exist increasing functions $s, r$ : $\mathbb{N} \rightarrow \mathbb{N}$ such that for all $i, j, n \in \mathbb{N}$ with $q_{i}\left(y_{n}\right) q_{j}\left(y_{n}\right)>0$ we have

$$
\frac{q_{i}\left(y_{n}\right)}{q_{r(j)}\left(y_{n}\right)} \leq \frac{p_{s(i)}\left(x_{n}\right)}{p_{s(j)}\left(x_{n}\right)} \leq \frac{q_{r(i)}\left(y_{n}\right)}{q_{j}\left(y_{n}\right)} .
$$

Proof. The sequence $\left(x_{n}\right)$ is equivalent to $\left(\alpha_{n} y_{n}\right)$ for some $\left(\alpha_{n}\right) \subset(\mathbb{K} \backslash\{0\})$. Thus there exists an isomorphism $T: \operatorname{lin}\left(x_{n}\right) \rightarrow \operatorname{lin}\left(y_{n}\right)$ with $T x_{n}=\alpha_{n} y_{n}, n \in \mathbb{N}$. By the continuity of $T$ and $T^{-1}$ we obtain

$$
\forall k \in \mathbb{N} \exists s(k), r(k) \in \mathbb{N} \forall x \in \operatorname{lin}\left(x_{n}\right): q_{k}(T x) \leq p_{s(k)}(x) \leq q_{r(k)}(T x) ;
$$

clearly, we can assume that $s(k)<s(k+1)$ and $r(k)<r(k+1)$ for any $k \in \mathbb{N}$. Then $\forall k, n \in \mathbb{N}: q_{k}\left(y_{n}\right) \leq p_{s(k)}\left(\alpha_{n}^{-1} x_{n}\right) \leq q_{r(k)}\left(y_{n}\right)$. Thus for all $k, n \in \mathbb{N}$ with $q_{k}\left(y_{n}\right)>0$ we have $\left[q_{k}\left(y_{n}\right) / p_{s(k)}\left(x_{n}\right)\right] \leq\left|\alpha_{n}^{-1}\right| \leq\left[q_{r(k)}\left(y_{n}\right) / p_{s(k)}\left(x_{n}\right)\right]$. Hence for all $i, j, n \in \mathbb{N}$ with $q_{i}\left(y_{n}\right) q_{j}\left(y_{n}\right)>0$ we get

$$
\frac{q_{i}\left(y_{n}\right)}{p_{s(i)}\left(x_{n}\right)} \leq \frac{q_{r(j)}\left(y_{n}\right)}{p_{s(j)}\left(x_{n}\right)} \text { and } \frac{q_{j}\left(y_{n}\right)}{p_{s(j)}\left(x_{n}\right)} \leq \frac{q_{r(i)}\left(y_{n}\right)}{p_{s(i)}\left(x_{n}\right)},
$$

or equivalently

$$
q_{i}\left(y_{n}\right) p_{s(j)}\left(x_{n}\right) \leq p_{s(i)}\left(x_{n}\right) q_{r(j)}\left(y_{n}\right) \text { and } q_{j}\left(y_{n}\right) p_{s(i)}\left(x_{n}\right) \leq p_{s(j)}\left(x_{n}\right) q_{r(i)}\left(y_{n}\right) .
$$

This implies Lemma 1.

Using this lemma, we shall show the following.

Theorem 2. Any non-normable Fréchet space $E$ with a semi-symmetric absolute basis $\left(e_{n}\right)$ is isomorphic to $\omega$. In particular, any non-normable Fréchet space with a symmetric absolute basis is isomorphic to $\omega$.

Proof. Let $\left(p_{k}\right)$ be a base of continuous seminorms on $E$. Suppose, by contradiction, that $E$ is not isomorphic to $\omega$. Then, for some $k \in \mathbb{N}$, the space $\left(E / \operatorname{ker} p_{k}\right)$ is infinite-dimensional. Thus the set $M_{1}=\left\{n \in \mathbb{N}: p_{k}\left(e_{n}\right)>0\right\}$ is infinite. Put $M_{2}=\left(\mathbb{N} \backslash M_{1}\right)$. Let $E_{i}$ be the closed linear span of $\left\{e_{n}: n \in M_{i}\right\}$ for $i=1,2$. Clearly, $p_{k}^{*} \mid E_{1}$ is a continuous norm on $E_{1}$ and $E$ is isomorphic to $E_{1} \times E_{2}$. We shall prove that $E$ has a continuous norm. It is obvious if $M_{2}$ is finite. Otherwise, there exists a permutation of $\mathbb{N}$ with $\pi\left(M_{2}\right)=M_{1}$. Since the basis $\left(e_{n}\right)$ is semi-symmetric, the sequence $\left(e_{\pi(n)}\right)$ is semi-equivalent to $\left(e_{n}\right)$. Then $\left(e_{\pi(n)}\right)_{n \in M_{2}}$ is semi-equivalent to $\left(e_{n}\right)_{n \in M_{2}}$. Thus $E_{1}$ is isomorphic to $E_{2}$. It follows that $E$ possesses a continuous norm. 
Since $E$ is non-normable, then for $M=\{2 n-1: n \in \mathbb{N}\}$ or $M=\{2 n: n \in \mathbb{N}\}$, the closed linear span $F$ of $\left\{e_{n}: n \in M\right\}$ is non-normable. Without loss of generality we can assume that $p_{k}^{*} \mid F, k \in \mathbb{N}$, are pairwise non-equivalent norms on $F$. Then $\lim \inf _{n \in M}\left[p_{k}\left(e_{n}\right) / p_{k+1}\left(e_{n}\right)\right]=0, k \in \mathbb{N}$. Let $\varphi: \mathbb{N}^{3} \rightarrow M$ be an injection. We can construct an injection $\psi: \mathbb{N}^{3} \rightarrow M$ such that

$$
\frac{p_{a}\left(e_{\psi(w)}\right)}{p_{a+1}\left(e_{\psi(w)}\right)}<\frac{p_{b}\left(e_{\varphi(w)}\right)}{p_{c}\left(e_{\varphi(w)}\right)} \text { for any } w=(a, b, c) \in \mathbb{N}^{3} .
$$

(We put the elements of the set $\mathbb{N}^{3}$ in a sequence $\left(t_{m}\right)$ and next we choose in turn $\psi\left(t_{1}\right), \psi\left(t_{2}\right), \ldots$.)

Since the maps $\varphi, \psi: \mathbb{N}^{3} \rightarrow M$ are injective and the sets $\left[\mathbb{N} \backslash \varphi\left(\mathbb{N}^{3}\right)\right],\left[\mathbb{N} \backslash \psi\left(\mathbb{N}^{3}\right)\right]$ are countable and infinite, there exists a permutation $\pi$ of $\mathbb{N}$ with $\pi(\varphi(w))=\psi(w)$ for all $w \in \mathbb{N}^{3}$. Then

$$
\forall(a, b, c) \in \mathbb{N}^{3} \exists n \in \mathbb{N}: \frac{p_{a}\left(e_{\pi(n)}\right)}{p_{a+1}\left(e_{\pi(n)}\right)}<\frac{p_{b}\left(e_{n}\right)}{p_{c}\left(e_{n}\right)} .
$$

Since $\left(e_{n}\right)$ is semi-symmetric, $\left(e_{\pi(n)}\right)$ is semi-equivalent to $\left(e_{n}\right)$. By Lemma 1 , there exist increasing functions $s, r: \mathbb{N} \rightarrow \mathbb{N}$ such that

$$
\forall i, j, n \in \mathbb{N}: \frac{p_{r(i)}\left(e_{\pi(n)}\right)}{p_{j}\left(e_{\pi(n)}\right)} \geq \frac{p_{s(i)}\left(e_{n}\right)}{p_{s(j)}\left(e_{n}\right)} .
$$

Hence, for $(a, b, c)=(r(1), s(1), s(r(1)+1))$, we have

$$
\forall n \in \mathbb{N}: \frac{p_{a}\left(e_{\pi(n)}\right)}{p_{a+1}\left(e_{\pi(n)}\right)} \geq \frac{p_{b}\left(e_{n}\right)}{p_{c}\left(e_{n}\right)} ;
$$

a contradiction. Thus $E$ is isomorphic to $\omega$.

For Fréchet-Schwartz spaces we shall show a stronger result.

Theorem 3. Any Fréchet-Schwartz space E with a semi-subsymmetric absolute basis $\left(e_{n}\right)$ is isomorphic to $\omega$. In particular, any Fréchet-Schwartz space with a subsymmetric absolute basis is isomorphic to $\omega$.

Proof. Let $\left(q_{k}\right)$ be a base of continuous seminorms on $E$. Suppose, by contradiction, that $E$ is not isomorphic to $\omega$. Then there is $i_{1} \in \mathbb{N}$ with $\operatorname{dim}\left(E / \operatorname{ker} q_{i_{1}}\right)=\infty$. Let $i \geq i_{1}$. Put $N_{i}=\left\{n \in \mathbb{N}: q_{i}\left(e_{n}\right)>0\right\}$. Clearly, the closed linear span $E_{i}$ of $\left\{e_{n}\right.$ : $\left.n \in N_{i}\right\}$ is an infinite-dimensional Fréchet-Schwartz space with an absolute basis $\left(e_{n}\right)_{n \in N_{i}}$ and $q_{i}^{*} \mid E_{i}$ is a continuous norm on $E_{i}$. Therefore $\lim _{n \in N_{i}}\left[q_{i}\left(e_{n}\right) / q_{j}\left(e_{n}\right)\right]=$ 0 for some $j>i$. Thus we can construct inductively an increasing sequence $\left(i_{k}\right) \subset \mathbb{N}$ such that $\lim _{n \in N_{i_{k}}}\left[q_{i_{k}}\left(e_{n}\right) / q_{i_{k+1}}\left(e_{n}\right)\right]=0$ for any $k \in \mathbb{N}$. Put $p_{k}=q_{i_{k}}$ and $M_{k}=$ $N_{i_{k}}$ for all $k \in \mathbb{N}$; obviously $M_{i} \subset M_{i+1}$ for any $i \in \mathbb{N}$. Let $a_{i, j}(n)=\left[p_{i}\left(e_{n}\right) / p_{j}\left(e_{n}\right)\right]$ for $i, j \in \mathbb{N}$ with $i<j$ and $n \in M_{i}$. Then $\lim _{n \in M_{i}} a_{i, j}(n)=0$ for all $i, j \in \mathbb{N}$ with $i<j$. Thus we can construct inductively an increasing sequence $\left(t_{n}\right) \subset M_{1}$ such that for any $n>1$ we have

$$
\begin{gathered}
\max _{1 \leq p<q \leq n} a_{p, q}\left(t_{n}\right)<\min _{1 \leq h<n} \min \left\{a_{h, n}(l): l \in M_{h}, l \leq b_{h, n}\right\} \\
\text { where } b_{h, n}=\max _{h \leq d<w \leq n} \max \left\{f \in M_{h}: a_{d, w}(f) \geq a_{1, n}\left(t_{n-1}\right)\right\} .
\end{gathered}
$$

(If $h \leq d<w \leq n$, then the set $\left\{f \in M_{h}: a_{d, w}(f) \geq a_{1, n}\left(t_{n-1}\right)\right\}$ is finite because $\lim _{f \in M_{h}} a_{d, w}(f)=0$. Moreover, $\lim _{t \in M_{1}} \max _{1 \leq p<q \leq n} a_{p, q}(t)=0$ for any $n \in \mathbb{N}$.) 
Let $\pi$ be a permutation of $\mathbb{N}$ with $\pi\left(M_{1}\right)=M_{1}$ and $\pi\left(t_{3 m}\right)=t_{3 m}, \pi\left(t_{3 m+1}\right)=$ $t_{3 m-1}$ for any $m \in \mathbb{N}$. Since the basis $\left(e_{n}\right)$ is semi-subsymmetric, the sequence $\left(e_{\pi(n)}\right)$ is semi-equivalent to $\left(e_{k_{n}}\right)$ for some increasing sequence $\left(k_{n}\right) \subset \mathbb{N}$. Then $\left(e_{\pi(n)}\right)_{n \in M_{1}}$ is semi-equivalent to $\left(e_{k_{n}}\right)_{n \in M_{1}}$. Thus the linear span $Y$ of $\left\{e_{k_{n}}: n \in\right.$ $\left.M_{1}\right\}$ is isomorphic to the linear span of $\left\{e_{n}: n \in M_{1}\right\}$; so $Y$ has a continuous norm. Therefore $\left\{k_{n}: n \in M_{1}\right\} \subset M_{h}$ for some $h \in \mathbb{N}$. Using Lemma 1 , we infer that there exist increasing functions $s, r: \mathbb{N} \rightarrow \mathbb{N}$ such that

$$
\frac{p_{i}\left(e_{k_{n}}\right)}{p_{r(j)}\left(e_{k_{n}}\right)} \leq \frac{p_{s(i)}\left(e_{\pi(n)}\right)}{p_{s(j)}\left(e_{\pi(n)}\right)} \leq \frac{p_{r(i)}\left(e_{k_{n}}\right)}{p_{j}\left(e_{k_{n}}\right)}
$$

for all $i, j, n \in \mathbb{N}$ with $p_{i}\left(e_{k_{n}}\right) p_{j}\left(e_{k_{n}}\right)>0$. Hence for $(i, j)=(h, r(h)+1)$ and $(u, v)=(h, r(r(h)+1)),(p, q)=(s(h), s(r(h)+1)),(d, w)=(r(h), r(h)+1)$ we get $a_{u, v}\left(k_{n}\right) \leq a_{p, q}(\pi(n)) \leq a_{d, w}\left(k_{n}\right)$ for any $n \in M_{1}$, since $p_{h}\left(e_{k_{n}}\right) p_{r(h)+1}\left(e_{k_{n}}\right)>0$ for any $n \in M_{1}$.

Let $m \in \mathbb{N}$ with $3 m \geq \max \{v, q, w\}$. Then $1 \leq h<3 m, 1 \leq p<q \leq 3 m, h \leq$ $d<w \leq 3 m, k_{t_{3 m}} \in M_{h}, k_{t_{3 m+1}} \in M_{h}, a_{p, q}\left(t_{3 m}\right)=a_{p, q}\left(\pi\left(t_{3 m}\right)\right) \geq a_{u, v}\left(k_{t_{3 m}}\right) \geq$ $a_{h, 3 m}\left(k_{t_{3 m}}\right)$ and $a_{d, w}\left(k_{t_{3 m+1}}\right) \geq a_{p, q}\left(\pi\left(t_{3 m+1}\right)\right)=a_{p, q}\left(t_{3 m-1}\right) \geq a_{1,3 m}\left(t_{3 m-1}\right)$.

By (1) we obtain $k_{t_{3 m}}>b_{h, 3 m}$ since $a_{p, q}\left(t_{3 m}\right) \geq a_{h, 3 m}\left(k_{t_{3 m}}\right)$ and $k_{t_{3 m}} \in M_{h}$. Moreover, $b_{h, 3 m} \geq k_{t_{3 m+1}}$ because $k_{t_{3 m+1}} \in M_{h}$ and $a_{d, w}\left(k_{t_{3 m+1}}\right) \geq a_{1,3 m}\left(t_{3 m-1}\right)$. Thus $k_{t_{3 m}}>k_{t_{3 m+1}}$. Hence $t_{3 m}>t_{3 m+1}$, so $3 m>3 m+1$; a contradiction. It follows that $E$ is isomorphic to $\omega$.

Corollary 4. Any nuclear Fréchet space with a semi-subsymmetric basis (in particular, with a subsymmetric basis) is isomorphic to $\omega$.

Now we show that there exist uncountably many mutually non-quasi-equivalent absolute subsymmetric bases in Fréchet spaces. Let $\mathcal{D}$ be the set of all sequences $a=\left(a_{n}\right) \subset(0, \infty)$ such that $\limsup _{n} \liminf _{m}\left|a_{n}-a_{m}\right|<\infty$. Let $a \in \mathcal{D}$. Put $B=\left(b_{n, k}\right)$ where $b_{n, k}=k^{a_{n}}$ for all $n, k \in \mathbb{N}$. Denote by $D_{\infty}(a)$ the Köthe space $K(B)$. We shall prove the following.

Proposition 5. (a) For any $a \in \mathcal{D}$ the coordinate basis in the Köthe space $D_{\infty}(a)$ is subsymmetric.

(b) There exists an uncountable family $\mathcal{D}^{\prime} \subset \mathcal{D}$ such that the coordinate bases in $D_{\infty}\left(a^{\prime}\right)$ and $D_{\infty}\left(a^{\prime \prime}\right)$ are not quasi-equivalent for any $a^{\prime}, a^{\prime \prime} \in \mathcal{D}^{\prime}$ with $a^{\prime} \neq a^{\prime \prime}$.

Proof. (a) Let $a=\left(a_{n}\right) \in \mathcal{D}$ and let $\left(e_{\pi(n)}\right)$ be a permutation of the coordinate basis $\left(e_{n}\right)$ in $D_{\infty}(a)$. By the definition of $\mathcal{D}$, there exist $c \in \mathbb{N}$ and an increasing sequence $\left(k_{n}\right) \subset \mathbb{N}$ such that $\left|a_{\pi(n)}-a_{k_{n}}\right| \leq(c-1), n \in \mathbb{N}$. Then

$$
\left(a_{\pi(n)}+1\right) \leq c\left(a_{k_{n}}+1\right) \leq c^{2}\left(a_{\pi(n)}+1\right), n \in \mathbb{N}
$$

Let $s(j)=j^{c}$ and $r(j)=j^{c^{2}}$ for each $j \in \mathbb{N}$ and $b_{n, k}=k^{a_{n}}$ for $n, k \in \mathbb{N}$. Then

$$
\forall j, n \in \mathbb{N}: j b_{\pi(n), j} \leq s(j) b_{k_{n}, s(j)} \leq r(j) b_{\pi(n), r(j)} .
$$

Consider a linear map $T: \operatorname{lin}\left(e_{\pi(n)}\right) \rightarrow \operatorname{lin}\left(e_{k_{n}}\right)$, such that $T e_{\pi(n)}=e_{k_{n}}$ for any $n \in \mathbb{N}$. By $(2)$ we get

$$
\forall j \in \mathbb{N} \forall x \in \operatorname{lin}\left(e_{\pi(n)}\right): p_{j}(x) \leq p_{s(j)}(T x) \leq p_{r(j)}(x),
$$

where $\left(p_{k}\right)$ is the standard base of continuous norms on $D_{\infty}(a)$. Therefore $T$ is an isomorphism. Thus $\left(e_{\pi(n)}\right)$ is equivalent to $\left(e_{k_{n}}\right)$, so $\left(e_{n}\right)$ is subsymmetric. 
(b) Let $\varphi: \mathbb{Q} \rightarrow \mathbb{N}$ be an injection. For $x \in \mathbb{R}$ let $\left(t_{x, n}\right) \subset(\mathbb{Q} \backslash\{x\})$ with $\lim t_{x, n}=x$ and $N_{x}=\left\{\varphi\left(t_{x, n}\right): n \in \mathbb{N}\right\}$. Then $\left\{N_{x}: x \in \mathbb{R}\right\}$ is an uncountable family of infinite subsets of $\mathbb{N}$ such that the set $N_{x} \cap N_{y}$ is finite for all $x, y \in \mathbb{R}$ with $x \neq y$.

Let $\left(h_{m}\right) \subset(1, \infty)$ be an increasing sequence with $\lim _{m} h_{2 m}^{-1} h_{2 m+1}=\infty$. Put $I_{m}=\left(h_{2 m-1}, h_{2 m}\right), m \in \mathbb{N}$. For $x \in \mathbb{R}$ let $a_{x}=\left(a_{x, n}\right)$ be a sequence with $\left\{a_{x, n}: n \in\right.$ $\mathbb{N}\}=\bigcup\left\{I_{m} \cap \mathbb{Q}: m \in N_{x}\right\}$. Clearly, $a_{x} \in \mathcal{D}$ for any $x \in \mathbb{R}$. Put $\mathcal{D}^{\prime}=\left\{a_{x}: x \in \mathbb{R}\right\}$. We shall prove that the coordinate bases in $D_{\infty}\left(a_{x}\right)$ and $D_{\infty}\left(a_{y}\right)$ are not quasiequivalent for any $x, y \in \mathbb{R}$ with $x \neq y$. Let $x, y \in \mathbb{R}$ with $x \neq y$.

Let $\pi$ be a permutation of $\mathbb{N}$. Take an increasing sequence $\left(d_{n}\right) \subset\left(N_{x} \backslash N_{y}\right)$ and a sequence $\left(s_{n}\right) \subset \mathbb{N}$ such that $a_{x, \pi\left(s_{n}\right)} \in I_{d_{n}}, n \in \mathbb{N}$. Then $\lim _{n} a_{x, \pi\left(s_{n}\right)}=\infty$ and

$$
\left(a_{x, \pi\left(s_{n}\right)}, a_{y, s_{n}}\right) \in\left[\left(\bigcup_{k=1}^{\infty} I_{k} \times \bigcup_{k=1}^{\infty} I_{k}\right) \backslash \bigcup_{k=1}^{\infty} I_{k} \times I_{k}\right], n \in \mathbb{N} .
$$

Thus $a_{x, \pi\left(s_{n}\right)}, a_{y, s_{n}} \in(1, \infty)$ for $n \in \mathbb{N}$ and

$$
\max \left\{\left(\frac{a_{x, \pi\left(s_{n}\right)}}{a_{y, s_{n}}}\right),\left(\frac{a_{y, s_{n}}}{a_{x, \pi\left(s_{n}\right)}}\right)\right\} \rightarrow_{n} \infty .
$$

Hence

because

$$
\max \left\{\left(\frac{a_{x, \pi\left(s_{n}\right)}+1}{a_{y, s_{n}}+1}\right),\left(\frac{a_{y, s_{n}}+1}{a_{x, \pi\left(s_{n}\right)}+1}\right)\right\} \rightarrow_{n} \infty
$$

$$
2\left(\frac{a_{x, \pi\left(s_{n}\right)}}{a_{y, s_{n}}}\right)>\left(\frac{a_{x, \pi\left(s_{n}\right)}+1}{a_{y, s_{n}}+1}\right)>\frac{1}{2}\left(\frac{a_{x, \pi\left(s_{n}\right)}}{a_{y, s_{n}}}\right), n \in \mathbb{N} .
$$

Suppose that the permutation $\left(e_{\pi(n)}\right)$ of the coordinate basis in $D_{\infty}\left(a_{x}\right)$ is semiequivalent to the coordinate basis $\left(e_{n}\right)$ in $D_{\infty}\left(a_{y}\right)$. Then by Lemma 1 there exist increasing functions $s, r: \mathbb{N} \rightarrow \mathbb{N}$ such that

$$
\forall i, j, n \in \mathbb{N}: \frac{p_{y, i}\left(e_{n}\right)}{p_{y, r(j)}\left(e_{n}\right)} \leq \frac{p_{x, s(i)}\left(e_{\pi(n)}\right)}{p_{x, s(j)}\left(e_{\pi(n)}\right)} \leq \frac{p_{y, r(i)}\left(e_{n}\right)}{p_{y, j}\left(e_{n}\right)}
$$

where $\left(p_{x, k}\right)$ and $\left(p_{y, k}\right)$ are the standard bases of continuous seminorms on $D_{\infty}\left(a_{x}\right)$ and $D_{\infty}\left(a_{y}\right)$, respectively. Thus

$$
\forall i, j, n \in \mathbb{N}:\left[\frac{i^{a_{y, n}+1}}{r(j)^{a_{y, n}+1}}\right] \leq\left[\frac{s(i)^{a_{x, \pi(n)}+1}}{s(j)^{a_{x, \pi(n)}+1}}\right] \leq\left[\frac{r(i)^{a_{y, n}+1}}{j^{a_{y, n}+1}}\right] .
$$

Hence, for all $i, j, n \in \mathbb{N}$, we obtain

$$
\left(a_{y, n}+1\right) \ln \left[\frac{i}{r(j)}\right] \leq\left(a_{x, \pi(n)}+1\right) \ln \left[\frac{s(i)}{s(j)}\right] \leq\left(a_{y, n}+1\right) \ln \left[\frac{r(i)}{j}\right] .
$$

Then for $(i, j)=(r(1)+1,1)$ we get

$$
\frac{\ln [i / r(j)]}{\ln [s(i) / s(j)]} \leq\left(\frac{a_{x, \pi(n)}+1}{a_{y, n}+1}\right) \leq \frac{\ln [r(i) / j]}{\ln [s(i) / s(j)]}, n \in \mathbb{N} .
$$

Thus

$$
\max \left\{\left(\frac{a_{x, \pi\left(s_{n}\right)}+1}{a_{y, s_{n}}+1}\right),\left(\frac{a_{y, s_{n}}+1}{a_{x, \pi\left(s_{n}\right)}+1}\right)\right\} \nrightarrow_{n} \infty
$$

a contradiction. This shows that the coordinate bases in $D_{\infty}\left(a_{x}\right)$ and $D_{\infty}\left(a_{y}\right)$ are not quasi-equivalent.

Finally, we prove the following result. 
Theorem 6. Any non-normable Fréchet space E with a quasi-homogeneous absolute basis $\left(e_{n}\right)$ is isomorphic to $\omega$.

Proof. Let $\left(q_{k}\right)$ be a base of continuous seminorms on $E$. Suppose, by contradiction, that $E$ is not isomorphic to $\omega$. Then for some $k \in \mathbb{N}$, we have $\operatorname{dim}\left(E / \operatorname{ker} q_{k}\right)=\infty$, so the set $L=\left\{n \in \mathbb{N}: q_{k}\left(e_{n}\right)>0\right\}$ is infinite. Denote by $X$ the closed linear span of $\left(e_{n}\right)_{n \in L}$; clearly, $q_{k}^{*} \mid X$ is a continuous norm on $X$. Since the basis $\left(e_{n}\right)$ is quasi-homogeneous, then $\left(e_{n}\right)_{n \in L}$ is quasi-equivalent to $\left(e_{n}\right)$; so $X$ is isomorphic to $E$. Thus $E$ has a continuous norm. Without loss of generality we can assume that $q_{1}$ is a norm.

Let $M$ be an infinite subset of $\mathbb{N}$. Since $\left(e_{n}\right)_{n \in M}$ is quasi-equivalent to $\left(e_{n}\right)$, the closed linear span $Y$ of $\left(e_{n}\right)_{n \in M}$ is isomorphic to $E$, so $Y$ is non-normable. Hence

$$
\forall k \in \mathbb{N} \exists l>k: \inf _{n \in M} \frac{q_{k}\left(e_{n}\right)}{q_{l}\left(e_{n}\right)}=0 .
$$

Thus we can construct inductively an increasing sequence $\left(k_{i}\right) \subset \mathbb{N}$ and a decreasing sequence $\left(M_{i}\right)$ of infinite subsets of $\mathbb{N}$ such that $\lim _{n \in M_{i}}\left[q_{k_{i}}\left(e_{n}\right) / q_{k_{i+1}}\left(e_{n}\right)\right]=0$ for any $i \in \mathbb{N}$. Put $p_{i}=q_{k_{i}}, i \in \mathbb{N}$. Then there exists an increasing sequence $\left(t_{n}\right) \subset \mathbb{N}$ such that

$$
\forall n \in \mathbb{N}: \min _{1 \leq v \leq n} \frac{p_{1}\left(e_{v}\right)}{p_{n}\left(e_{v}\right)}>\max _{1 \leq i \leq n} \frac{p_{i}\left(e_{t_{n}}\right)}{p_{i+1}\left(e_{t_{n}}\right)} .
$$

Since $\left(e_{t_{n}}\right)$ is quasi-equivalent to $\left(e_{n}\right)$, there is a permutation $\pi$ of $\mathbb{N}$ such that $\left(e_{\pi(n)}\right)$ is semi-equivalent to $\left(e_{t_{n}}\right)$. By Lemma 1, there exist increasing functions $s, r: \mathbb{N} \rightarrow \mathbb{N}$ such that

$$
\forall i, j, n \in \mathbb{N}: \frac{p_{s(i)}\left(e_{\pi(n)}\right)}{p_{s(j)}\left(e_{\pi(n)}\right)} \leq \frac{p_{r(i)}\left(e_{t_{n}}\right)}{p_{j}\left(e_{t_{n}}\right)} .
$$

In particular, for $(a, b, c)=(r(1), s(1), s(r(1)+1))$ we have

$$
\forall n \in \mathbb{N}: \frac{p_{b}\left(e_{\pi(n)}\right)}{p_{c}\left(e_{\pi(n)}\right)} \leq \frac{p_{a}\left(e_{t_{n}}\right)}{p_{a+1}\left(e_{t_{n}}\right)} .
$$

Using (3), we get $\pi(n)>n$ for any $n \geq c$ because

$$
\frac{p_{1}\left(e_{\pi(n)}\right)}{p_{n}\left(e_{\pi(n)}\right)} \leq \frac{p_{b}\left(e_{\pi(n)}\right)}{p_{c}\left(e_{\pi(n)}\right)}
$$

and $a \leq n$ for any $n \geq c$. Hence $\pi(\{i \in \mathbb{N}: i \geq c\}) \subset\{i \in \mathbb{N}: i>c\}$, so $\pi(\{i \in \mathbb{N}: i<c\}) \supset\{i \in \mathbb{N}: i \leq c\}$; this is impossible.

Thus $E$ is isomorphic to $\omega$.

Corollary 7. Any nuclear Fréchet space with a quasi-homogeneous basis (in particular, with a homogeneous one) is isomorphic to $\omega$.

\section{REFERENCES}

[1] Bessaga C., Pełczyński A., On a class of $B_{0}$-spaces, Bull. Acad. Pol. Sci., 5(1957), 357-377. MR0088680 (19:562b)

[2] Bessaga C., Pełczyński A., Własności baz w przestrzeniach typu $B_{0}$, (in Polish), Prace Matematyczne 3(1959), 123-142. MR0126691 (23:A3986)

[3] Cac N. P., On symmetric Schauder bases in Fréchet spaces, Studia Math. 32(1969), 95-98. MR0243310(39:4632)

[4] Garling D.J.H., Symmetric bases of locally convex spaces, Studia Math. 30(1968), 163-181. MR0230105 (37:5670) 
[5] Rolewicz S., Metric linear spaces, PWN-Polish Scientific Publishers - Warszawa, 1984. MR.0802450 (88i:46004a)

[6] Ruckle W., Symmetric coordinate spaces and symmetric bases, Canadian J. Math. 19(1967), 828-839. MR0236682 (38:4977)

[7] Ruckle W., On the characterization of sequence spaces associated with Schauder bases, Studia Math. 28(1967), 279-288. MR0215049 (35:5894)

[8] Singer I., Bases in Banach spaces I, Springer-Verlag Berlin - Heidelberg - New York, 1970. MR0298399 (45:7451)

[9] Terzioğlu T., Symmetric bases of nuclear spaces, J. Reine Angew. Math. 252(1972), 200-204. MR 0291758(45:849)

Faculty of Mathematics and Computer Science, Adam Mickiewicz University, ul. Umultowska 87, 61-614 Poznań, Poland

E-mail address: sliwa@amu.edu.pl 